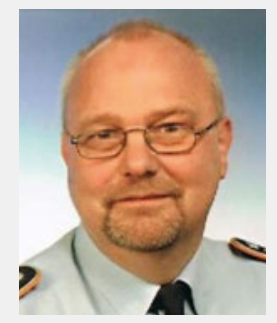

Dr. Torsten Pippig

\section{Ist die aktuelle Flugmedizin wissenschaftlich genug?}

\author{
Liebe Kolleginnen und Kollegen,
}

die Flugmedizin ist eine Teildisziplin der Medizin, die sich mit der Luftfahrt- und Raumfahrtmedizin befasst. Gegenstand der Flugmedizin sind die physikalischen und medizinischen Besonderheiten des Aufenthalts in Luft- und Weltraum sowie das Wohlergehen des fliegenden Personals und der Passagiere. Die 4 tragenden Säulen sind die fliegerärztliche Begutachtung, die fachliche Aus- und Weiterbildung der Fliegerärzte, die Beratung der Passagiere (einschließlich der Lufttransport Verletzter und Erkrankter) und die Wissenschaft. Die Teilnahme am Wissenschaftsbetrieb ist grundsätzlich nicht an Voraussetzungen oder Bedingungen geknüpft, die wissenschaftliche Betätigung außerhalb des akademischen oder industriellen Wissenschaftsbetriebs steht jedermann offen und ist auch gesetzlich von der Forschungsfreiheit abgedeckt. Somit werden die individuellen wissenschaftlichen Fähigkeiten, Fertigkeiten und Aktivitäten durch die persönliche Einsicht in die Notwendigkeit „sich wissenschaflich zu betätigen" des Einzelnen bestimmt. Das wissenschaftliche Interesse und die Kompetenz des Einzelnen kann man an der Anzahl und Qualität seiner Vorträge und Publikationen, an der Teilnahme an wissenschaftlichen Kongressen und Symposien messen.

Wie kann man nun die „Wissenschaftlichkeit“ der aktuellen Flugmedizin messen? Eine Bewertungsmöglichkeit ist die Organisation der Fliegerärzte (und aller Freunde der Flugmedizin) in „ihrer“ Fachgesellschaft der DGLRM e.V. (derzeit ca. 430 Mitglieder) und deren Aktivitäten. Eine wichtige Kennzahl ist neben der Teilnehmerzahl an der wissenschaftlichen Jahrestagung auch die Quantität und Qualität der Präsentationen und Fachvorträge, diesmal die 55. Jahrestagung, vom 14. bis 17. September 2017 in Köln. Des Weiteren bietet die DGLRM e.V. die Mitarbeit in verschiedenen Arbeitsgruppen an, in den folgenden Ausgaben der FTR wird immer eine AG vorgestellt (siehe auch www. dglrm.de). Wenn Sie etwas finden, dann melden Sie sich beim AG-Leiter. Ein weiterer wichtiger Marker sind die Anzahl und das Niveau der Publikationen in unserer Fachzeitschrift der Fachgesellschaft, in der Flugmedizin Tropenmedizin und Reisemedizin, welche einmal im Jahr mit dem Leitthema „Flugmedizin“ erscheint, in dieser Ausgabe. Ich fordere Sie alle auf, kommen Sie zu unserer 55. wissenschaftlichen Jahrestagung in Köln, diskutieren Sie kritisch und offen mit uns, bewerten Sie die Qualität der Fachvorträge und Poster. Auch breche ich wieder eine Lanze für die FTR, die 2017 neu gestaltet wurde. Bewerten Sie die Artikel, diskutieren Sie mit den Autoren. Nur der ständige Kontakt und Austausch bringt uns wirklich voran. Geben Sie uns Tipps und Ratschläge wie wir unsere Fachgesellschaft und unsere Fachzeitschrift weiterentwickeln können.

In dieser Ausgabe werden 4 flugmedizinische Publikationen präsentiert, die das breite Betätigungsfeld der Flugmedizin repräsentieren. Herr Siedenburg und Herr Knöffler schreiben über flugmedizinische und gutachterlich relevante Themen, über die Flugangst beziehungsweise über das Akustikusneurinom, Herr Kalina und Herr Hinkelbein beschreiben das unterschiedliche notfallmedizinische Equipment in europäischen Verkehrsflugzeugen und fordern eine standardisierte Versorgung und Vereinheitlichung der notfallmedizinischen Ausstattung. Herr Oehler (Rechtsanwalt), befasst sich mit einem sehr wichtigen Thema für uns Ärzte, der ärztlichen Schweigepflicht. Gerade nach der Katastrophe vom 24. März 2015 und den nachfolgenden Untersuchungen, Diskussionen, Bewertungen, Forderungen und Empfehlungen ist ein klares Ja zur ärztlichen Schweigepflicht für uns Ärzte und das Vertrauen der Piloten in uns Fliegerärzte wichtig.

Abschließend bitte ich sie alle, wagen Sie den fachlichen Kommentar, seien sie kritisch mit den Autoren und dem Geschriebenen, auch eine positive Bewertung ist Ansporn für die Autoren. 